\title{
DETERMINAÇÃO DE FATORES DE EMPILHAMENTO ATRAVÉS DE FOTOGRAFIAS DIGITAIS ${ }^{1}$
}

\author{
Carlos Pedro Boechat Soares², José Carlos Ribeiro², Moacir Batista do Nascimento Filho ${ }^{3}$ e \\ José Carlos Lopes Ribeiro ${ }^{4}$
}

\begin{abstract}
RESUMO - O objetivo deste trabalho foi analisar a exatidão de estimativas de fatores de empilhamento obtidas através de fotografias digitais e de software especialmente desenvolvido para esta finalidade, em pilhas sobre caminhões. Para isto, foram utilizados seis caminhões com cinco pilhas cada um, pertencentes à ACESITA ENERGÉTICA Ltda. Após as análises, verificou-se que a diferença média entre os fatores obtidos pelo método fotográfico e os fatores reais foi da ordem de 3,2\%, com tendência de superestimação dos fatores obtidos pelo método fotográfico. Pelo teste " $t$ ”, verificou-se, também, que os fatores obtidos pelo método fotográfico diferiram dos fatores reais, principalmente devido ao mau empilhamento da madeira.
\end{abstract}

Palavras-chave: Fatores de empilhamento, fotografia digital e caminhões.

\section{DETERMINATION OF PILING FACTORS THROUGH DIGITAL PHOTOGRAPHY}

\begin{abstract}
The objective of this work was to analyze the accuracy of piling factor estimates using digital photography and software especially developed for this purpose. Six trucks carrying five piles each, owned by ACESITA ENERGÉTICA Ltd., were used in this research. The analysis revealed that the average difference between the factors determined by the photographic method and the actual factors was approximately $3.2 \%$, with a tendency for over estimation of the values obtained by the photographic method. The " $t$ " test analysis suggested that the differences observed between these factors were mainly due to the the poor piling up of the wood.
\end{abstract}

Key words: $\quad$ Piling factors, digital photography and trucks.

\section{INTRODUÇÃO}

Há algum tempo, profissionais ligados à área de mensuração e grande parte das empresas florestais brasileiras vêm se preocupando com a determinação do volume de pilhas de madeira. Estimativas imprecisas do volume real de madeira das pilhas acarretam resultados distorcidos quanto aos rendimentos de processos de produção e quanto ao pagamento de serviços relacionados ao corte e transporte da madeira, bem como problemas na venda da madeira. Além disto, em muitos casos os volumes das pilhas de madeira são expressos em estéreo
- unidade muito comum na comercialização de madeira. No entanto, essa unidade não pertence ao Sistema Internacional de Unidades, implicando problemas legais para os contratos de prestação de serviços ou de compra e venda da madeira.

Embora o volume sólido de uma pilha de madeira possa ser obtido através do somatório dos volumes dos toros individualmente, este procedimento envolve um grande esforço de mão-de-obra e de tempo para sua realização. Assim sendo, a maneira mais usual de estimar o volume sólido de uma pilha de madeira é através da

1 Recebido para publicação em 24.9.2001.

Aceito para publicação em 9.9.2003.

2 Professores do Dep. de Engenharia Florestal da Universidade Federal de Viçosa - UFV, <csoares@mail.ufv.br> e <rib@mail.ufv.br>; ${ }^{3}$ Eng. Florestal da ACESITA ENERGÉTICA Ltda. - Pça 1º de Maio, 09, Centro, 35180-000 Timóteo-MG;

${ }^{4}$ Engenheiro Civil. 
medição das suas dimensões, com as quais se obtém o volume em estéreo, que multiplicado por um fator de conversão médio, denominado de fator de empilhamento, fornece o volume sólido de madeira. No entanto, características como os diâmetros e o comprimento dos toros de madeira, a presença ou não de casca, a maneira de empilhar a madeira, a espécie e a idade das árvores afetam o empilhamento, de tal forma que a utilização de um fator médio, obtido em uma condição diferente daquela que se trabalha, pode acarretar erro na estimação do volume sólido da pilha (Paula Neto \& Rezende, 1992). Como complicador, muitas vezes a determinação do volume sólido de madeira é feita diretamente em pilhas de madeira sobre a carroceria de caminhões, acarretando erros ainda maiores, devido à disposição dos toros sobre as carrocerias e à falta de uma boa visualização dos limites das faces das pilhas.

Portanto, percebe-se que metodologias confiáveis, precisas e de baixo custo devem ser desenvolvidas com o objetivo de agilizar a obtenção de estimativas do volume sólido de pilhas de madeira, de forma que estas estimativas possam ser utilizadas com segurança em processos produtivos e na comercialização da madeira. Neste sentido, vários trabalhos, dentre eles os de Moutain (1949), Keepers (1945), Rubio (1982) e Rezende (1988), evidenciam que através de fotografias, obtidas com uma câmera de $35 \mathrm{~mm}$, pode-se obter com precisão os fatores de empilhamento e o volume sólido de madeira em qualquer pilha sobre o solo. Garland (1968) mostrou, ainda, que o método fotográfico podia ser aplicado para determinação do volume sólido de madeira de pilhas sobre caminhões, com erro médio da ordem de $\pm 3 \%$. Além

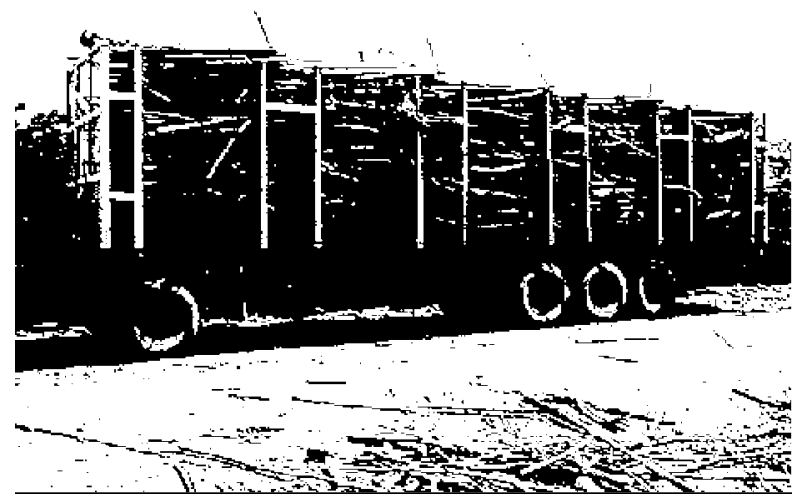

Figura 1 - Caminhão utilizado no transporte da madeira. Figure 1 - Truck used in the transport of the wood.

R. Árvore, Viçosa-MG, v.27, n.4, p.473-479, 2003 disto, outras metodologias foram desenvolvidas para determinação de fatores de empilhamento, as quais se baseiam no uso de câmeras filmadoras (Loetch et al., 1973), com uma precisão de $\pm 20 \%$, e no processamento de imagens digitais por meio de computadores - Sistema TOROS (Carvalho et al., 1992).

Diante do exposto, realizou-se este trabalho com o objetivo de analisar a exatidão de estimativas de fatores de empilhamento obtidos através de fotografias digitais e de software especialmente desenvolvido para esta finalidade, em pilhas sobre caminhões.

\section{MATERIAL E MÉTODOS}

O presente trabalho foi executado em área pertencente à ACESITA ENERGÉTICA Ltda., localizada no município de Capelinha, Minas Gerais, especificamente em um dos pátios de carvoejamento da empresa. Foram selecionados, aleatoriamente, seis caminhões que transportavam cinco pilhas de madeira de eucalipto cada, no sentido longitudinal à carroceria (Figura 1).

Foram tomadas as medidas de cinco alturas $\left(\mathrm{A}_{\mathrm{i}}\right) \mathrm{e}$ de três larguras $\left(\mathrm{L}_{\mathrm{i}}\right)$ em cada pilha do caminhão, com o objetivo de obter a área visível de cada face da pilha, pela multiplicação das dimensões médias (Figura 2). O volume em estéreo de cada pilha foi obtido ao multiplicar a área visível de cada pilha pelo comprimento médio dos

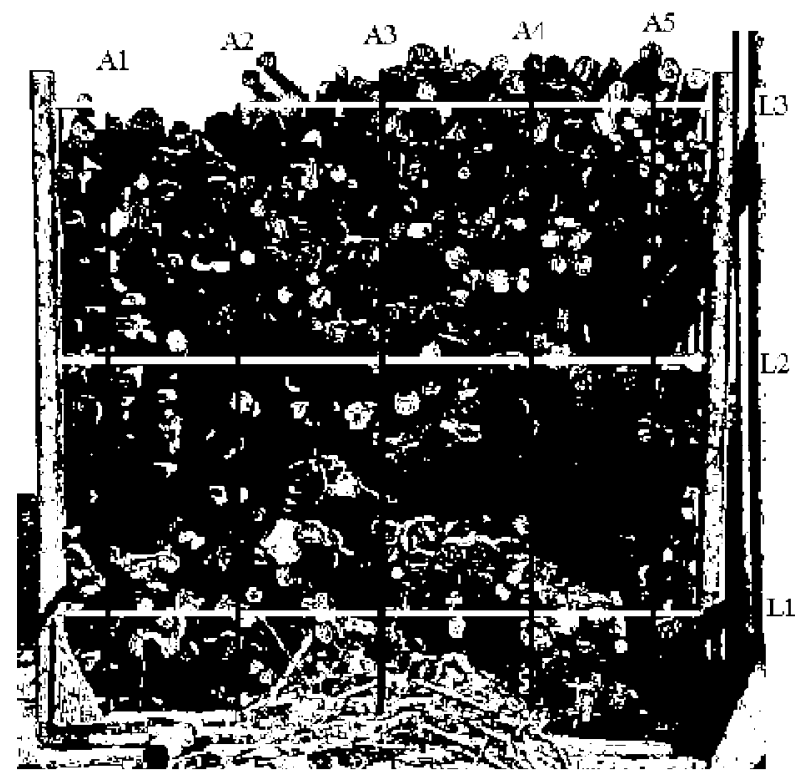

Figura 2 - Tomada de dimensões das pilhas nos caminhões. Figure 2 - Determination of pile dimension in the trucks. 
toros da pilha, obtido através da cubagem rigorosa, em uma etapa posterior. Em seguida, o volume em estéreo de cada caminhão foi obtido pela soma dos volumes em estéreo de cada pilha.

A face visível de cada pilha do caminhão foi identificada e fotografada a uma distância igual ao comprimento da carroceria, com uma câmera fotográfica digital SONY, modelo FD-31 (Mavica). As fotografias armazenadas pela câmera, em disquete, foram transferidas para o computador e analisadas através do software DIGITORA, para obtenção do fator de empilhamento com casca em cada pilha e do fator de empilhamento médio em cada caminhão.

A metodologia utilizada no DIGITORA é a mesma descrita por Husch et al. (1972), porém as fotos não precisaram ser reveladas e nem houve a necessidade da elaboração de um templet de forma manual. Através de um comando do software, uma rede de aproximadamente 200 pontos, distantes entre si 20 pixels na horizontal e 15 pixels na vertical, cobrindo cerca de $50 \%$ da área da foto, foi exibida aleatoriamente sobre a face da pilha. Assim sendo, cada ponto foi observado e classificado como:

- Ponto vazio: ponto projetado no espaço vazio entre as toras.

- Ponto meio: ponto projetado metade sobre a tora e metade no espaço vazio.

- Ponto anulado: ponto sobre área de difícil visualização.

Uma vez classificados os pontos sobre a fotografia, o software forneceu a porcentagem de área ocupada por madeira na face da pilha, ou seja, o fator de empilhamento com casca, através da seguinte expressão:

$$
\operatorname{Pm}(\%)=\left(1-\frac{\text { vazios }+\frac{\text { meio }}{2}}{\text { total }- \text { anulados }}\right)
$$

Cada toro foi cubado rigorosamente pela aplicação da expressão de Smalian (Husch et al., 1972), para obter o volume sólido de madeira em cada pilha e, conseqüentemente, o volume sólido de madeira em cada caminhão. Com o volume sólido e o volume em estéreo de cada pilha, calculou-se o fator de empilhamento real com casca, pela seguinte expressão:

$$
F e=\frac{V_{m^{3}}}{V_{s t}}
$$

em que

$F e=$ fator de empilhamento com casca;

$V_{m^{3}}=$ volume sólido de madeira na pilha, $\mathrm{em} \mathrm{m}^{3}$; e

$V_{s t}=$ volume de madeira empilhada, em st.

A exatidão do método fotográfico foi verificada pelo cálculo das diferenças porcentuais entre os fatores reais e os estimados pela fotografia e pela análise gráfica da distribuição das diferenças porcentuais por pilha e por caminhão. A precisão do método foi verificada pelo cálculo da média dos desvios porcentuais (MDP). Além disto verificou-se, pela aplicação do teste " $\mathrm{t}$ " para dados pareados, se os fatores obtidos pelo método fotográfico diferiam estatisticamente, a 95\% de probabilidade, dos fatores reais.

\section{RESULTADOS E DISCUSSÃO}

Analisando os resultados do Quadro 1, verificou-se que os caminhões transportavam, em média, 66,12 st de madeira, o que corresponde a $38,99 \mathrm{~m}^{3}$. O fator de empilhamento médio real com casca, referente às 30 pilhas de madeira, foi igual a 0,5909, enquanto o fator de empilhamento médio estimado pelo procedimento fotográfico foi igual a 0,6083, ou seja, o fator de empilhamento médio estimado pela fotografia foi maior que o fator médio real na ordem de $2,94 \%$. A média das diferenças porcentuais (MDP) foi igual a 3,23\%. As diferenças para as pilhas chegaram à ordem de até $15,56 \%$ (pilha 5, caminhão 1) e, para os caminhões, até $8,52 \%$ (caminhão 1 ), com uma tendência de superestimação dos volumes pelo procedimento fotográfico, que pode ser mais bem visualizada nas Figuras 3 e 4.

Pela aplicação do teste "t" para dados pareados, verificou-se (Quadro 2) que existe diferença significativa, a $95 \%$ de probabilidade, entre as estimativas dos fatores de empilhamento reais e os obtidos pelas fotografias. Cabe apenas salientar que o valor calculado da estatística " $t$ " ficou bem próximo do ponto crítico $(2,045)$, considerando o nível de probabilidade adotado.

Analisando os dados do Quadro 1 e a Figura 4, verificou-se que os caminhões 1 e 4 tiveram forte influência sobre o valor da estatística " $t$ ", haja vista que as diferenças entre os fatores obtidos para estes caminhões foram grandes. Procedendo-se a uma análise mais criteriosa das

R. Árvore, Viçosa-MG, v.27, n.4, p.473-479, 2003 
Quadro 1 - Volume em estéreo e em metro cúbico de cada pilha e de cada caminhão, fatores de empilhamento reais e estimados por fotografias, e diferenças porcentuais entre os fatores reais e estimados pelas fotografias

Table 1 - Estereo and cubic meter of each pile and truck, actual and estimated piling factors, and percentile differences between real and estimated piling factors

\begin{tabular}{|c|c|c|c|c|c|c|}
\hline $\begin{array}{c}\text { Caminhão } \\
\left(\mathrm{n}^{\circ}\right)\end{array}$ & Pilha & $\begin{array}{l}\text { Volume } \\
\text { (st) Real }\end{array}$ & $\begin{array}{l}\text { Volume } \\
\left(\mathrm{m}^{3}\right) \text { Real }\end{array}$ & $\begin{array}{l}\text { Fator } \\
\text { Real }\end{array}$ & $\begin{array}{c}\text { Fator } \\
\text { Fotografia }\end{array}$ & Diferença $(\%) *$ \\
\hline \multirow{5}{*}{$1(5221)$} & 1 & 13,1150 & 7,2263 & 0,5510 & 0,6024 & 9,3291 \\
\hline & 2 & 14,2236 & 8,0200 & 0,5638 & 0,6053 & 7,3514 \\
\hline & 3 & 14,1530 & 7,8156 & 0,5522 & 0,5690 & 3,0391 \\
\hline & 4 & 13,6602 & 7,5626 & 0,5536 & 0,5942 & 7,3286 \\
\hline & 5 & 14,2165 & 7,6149 & 0,5356 & 0,6190 & 15,5631 \\
\hline \multicolumn{2}{|c|}{ Soma / Média } & 69,3684 & 38,2394 & $\mathbf{0 , 5 5 1 3}$ & 0,5980 & 8,5223 \\
\hline \multirow{5}{*}{$2(5208)$} & 1 & 11,9651 & 6,6666 & 0,5572 & 0,6095 & 9,3916 \\
\hline & 2 & 12,5798 & 7,5415 & 0,5995 & 0,6205 & 3,5048 \\
\hline & 3 & 12,1533 & 7,6352 & 0,6282 & 0,5944 & $-5,3869$ \\
\hline & 4 & 11,9441 & 7,7985 & 0,6529 & 0,6275 & $-3,8933$ \\
\hline & 5 & 12,3099 & 7,8581 & 0,6384 & 0,6191 & $-3,0166$ \\
\hline \multicolumn{2}{|c|}{ Soma / Média } & 60,9521 & 37,4999 & 0,6152 & 0,6142 & 0,1199 \\
\hline \multirow{5}{*}{$3(6238)$} & 1 & 13,0206 & 7,2736 & 0,5586 & 0,6044 & 8,1946 \\
\hline & 2 & 13,4820 & 8,2279 & 0,6103 & 0,6024 & $-1,2923$ \\
\hline & 3 & 12,9106 & 7,8584 & 0,6087 & 0,6418 & 5,4416 \\
\hline & 4 & 13,5620 & 7,9737 & 0,5879 & 0,6029 & 2,5443 \\
\hline & 5 & 13,0448 & 8,2014 & 0,6287 & 0,6024 & $-4,1849$ \\
\hline \multicolumn{2}{|c|}{ Soma / Média } & 66,0199 & 39,5349 & $\mathbf{0 , 5 9 8 8}$ & 0,6108 & 2,1406 \\
\hline \multirow{5}{*}{$4(5218)$} & 1 & 13,7434 & 8,0956 & 0,5891 & 0,6063 & 2,9280 \\
\hline & 2 & 13,5167 & 7,7671 & 0,5746 & 0,5686 & $-1,0498$ \\
\hline & 3 & 14,7649 & 8,2040 & 0,5556 & 0,6256 & 12,5902 \\
\hline & 4 & 14,4868 & 7,9805 & 0,5509 & 0,6044 & 9,7159 \\
\hline & 5 & 14,5115 & 7,8556 & 0,5413 & 0,6048 & 11,7234 \\
\hline \multicolumn{2}{|c|}{ Soma / Média } & 71,0234 & 39,9028 & $\mathbf{0 , 5 6 1 8}$ & 0,6019 & 7,1816 \\
\hline \multirow{5}{*}{$5(5209)$} & 1 & 13,1269 & 8,2897 & 0,6315 & 0,6220 & $-1,5053$ \\
\hline & 2 & 12,9552 & 8,0701 & 0,6229 & 0,5833 & $-6,3611$ \\
\hline & 3 & 12,9633 & 7,7510 & 0,5979 & 0,6184 & 3,4259 \\
\hline & 4 & 11,7398 & 6,9091 & 0,5885 & 0,5934 & 0,8285 \\
\hline & 5 & 12,4601 & 7,4406 & 0,5972 & 0,6262 & 4,8648 \\
\hline \multicolumn{2}{|c|}{ Soma / Média } & 63,2452 & 38,4605 & 0,6081 & 0,6087 & 0,2506 \\
\hline \multirow{5}{*}{$6(5228)$} & 1 & 13,2507 & 8,5313 & 0,6438 & 0,6119 & $-4,9605$ \\
\hline & 2 & 12,7816 & 7,9269 & 0,6202 & 0,6097 & $-1,6894$ \\
\hline & 3 & 13,4992 & 8,0093 & 0,5933 & 0,6071 & 2,3236 \\
\hline & 4 & 13,1293 & 7,9139 & 0,6028 & 0,6256 & 3,7890 \\
\hline & 5 & 13,4576 & 7,9499 & 0,5907 & 0,6276 & 6,2398 \\
\hline \multicolumn{2}{|c|}{ Soma / Média } & 66,1184 & 40,3312 & 0,6100 & 0,6164 & 1,1405 \\
\hline \multicolumn{2}{|c|}{ Media Geral => } & 66,1212 & 38,9948 & $\mathbf{0 , 5 9 0 9}$ & 0,6083 & 3,2259 \\
\hline
\end{tabular}

* Diferença $(\%)=($ fotografia-real $) /$ real $* 100$. 


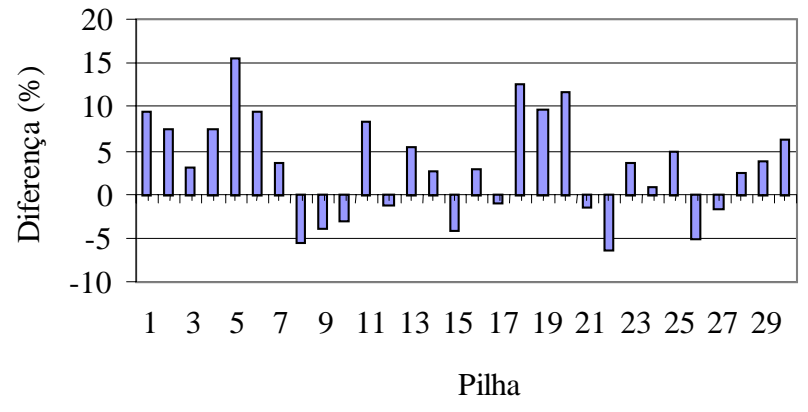

Figura 3 - Diferenças porcentuais por pilha.

Figure 3 - Percentile difference per pile.

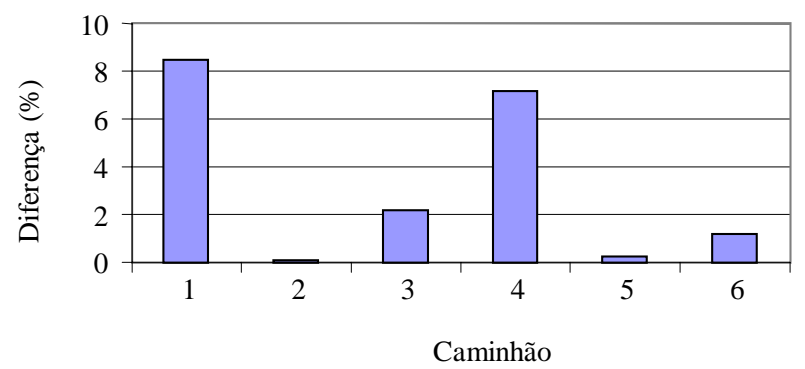

Figura 4 - Diferenças porcentuais por caminhão. Figure 4 - Percentile difference per truck.

fotografias das pilhas de madeira sobre os caminhões $1 \mathrm{e}$ 4 (Figuras 5 e 6), constatou-se que a qualidade do empilhamento mecânico da madeira acarretou dificuldades de análise das fotografias, refletindo em uma maior diferença entre os fatores para estes caminhões.

Assim sendo, para os caminhões 1 e 4, realizou-se um teste no qual foram feitas seis repetições por pilha, com o objetivo de verificar o efeito do número de repetições sobre a precisão das estimativas dos fatores de empilhamento. Os fatores de empilhamento médios e as diferenças porcentuais médias entre os volumes estão apresentados no Quadro 3.

Analisando os resultados do Quadro 3, verificou-se que o fator de empilhamento obtido por repetições (seis para cada pilha) não acarretou melhoria da precisão do método fotográfico, tendo em vista o aumento da diferença porcentual média entre as estimativas dos fatores. Desta forma, os fatores obtidos pelo procedimento fotográfico continuam diferindo estatisticamente dos fatores reais. Porém, como os seis caminhões foram selecionados aleatoriamente, cargas de madeira como as dos caminhões 1 e 4 são uma realidade da empresa. Assim, para que o procedimento fotográfico possa ser utilizado com maior precisão, há a necessidade de melhorar o empilhamento da madeira sobre os caminhões, bem como uniformizar o comprimento dos toros, que apresentaram, segundo a cubagem rigorosa, comprimentos inferiores a 1,0 m, causando problemas no empilhamento, conforme apresentado nas Figuras 5 e 6.

Quadro 2 - Diferenças entre as estimativas dos fatores de empilhamento reais (Real) e as estimativas obtidas pelo procedimento fotográfico e estatística "t" para dados pareados

Table 2 - Differences between actual and estimated piling factors; and statistic " $t$ " for dependent samples

\begin{tabular}{|c|c|c|c|}
\hline \multirow{2}{*}{$\begin{array}{l}\text { Pilha } \\
\mathrm{N}^{\mathrm{o}}\end{array}$} & \multicolumn{2}{|c|}{ Fator } & \multirow{2}{*}{$\begin{array}{c}\text { Diferença } \\
\text { Real-Fotografia }\end{array}$} \\
\hline & Real & Fotografia & \\
\hline 1 & 0,5510 & 0,6024 & $-0,0514$ \\
\hline 2 & 0,5638 & 0,6053 & $-0,0415$ \\
\hline 3 & 0,5522 & 0,5690 & $-0,0168$ \\
\hline 4 & 0,5536 & 0,5942 & $-0,0406$ \\
\hline 5 & 0,5356 & 0,6190 & $-0,0834$ \\
\hline 6 & 0,5572 & 0,6095 & $-0,0523$ \\
\hline 7 & 0,5995 & 0,6205 & $-0,0210$ \\
\hline 8 & 0,6282 & 0,5944 & 0,0338 \\
\hline 9 & 0,6529 & 0,6275 & 0,0254 \\
\hline 10 & 0,6384 & 0,6191 & 0,0193 \\
\hline 11 & 0,5586 & 0,6044 & $-0,0458$ \\
\hline 12 & 0,6103 & 0,6024 & 0,0079 \\
\hline 13 & 0,6087 & 0,6418 & $-0,0331$ \\
\hline 14 & 0,5879 & 0,6029 & $-0,0150$ \\
\hline 15 & 0,6287 & 0,6024 & 0,0263 \\
\hline 16 & 0,5891 & 0,6063 & $-0,0172$ \\
\hline 17 & 0,5746 & 0,5686 & 0,0060 \\
\hline 18 & 0,5556 & 0,6256 & $-0,0700$ \\
\hline 19 & 0,5509 & 0,6044 & $-0,0535$ \\
\hline 20 & 0,5413 & 0,6048 & $-0,0635$ \\
\hline 21 & 0,6315 & 0,6220 & 0,0095 \\
\hline 22 & 0,6229 & 0,5833 & 0,0396 \\
\hline 23 & 0,5979 & 0,6184 & $-0,0205$ \\
\hline 24 & 0,5885 & 0,5934 & $-0,0049$ \\
\hline 25 & 0,5972 & 0,6262 & $-0,0290$ \\
\hline 26 & 0,6438 & 0,6119 & 0,0319 \\
\hline 27 & 0,6202 & 0,6097 & 0,0105 \\
\hline 28 & 0,5933 & 0,6071 & $-0,0138$ \\
\hline 29 & 0,6028 & 0,6256 & $-0,0228$ \\
\hline 30 & 0,5907 & 0,6276 & $-0,0369$ \\
\hline \multirow{5}{*}{\multicolumn{2}{|c|}{$\begin{array}{l}\text { Resultado do teste } \\
\text { "tt" } \Rightarrow>\end{array}$}} & Média $=>$ & $-0,0174$ \\
\hline & & Desvio $=>$ & 0,0332 \\
\hline & & & 30 \\
\hline & & “t” calculado $=>$ & $-2,88 *$ \\
\hline & & “t” $(5 \%, 29 \mathrm{gl})=>$ & 2,045 \\
\hline
\end{tabular}

* significativo a $95 \%$ de probabilidade.

R. Árvore, Viçosa-MG, v.27, n.4, p.473-479, 2003 


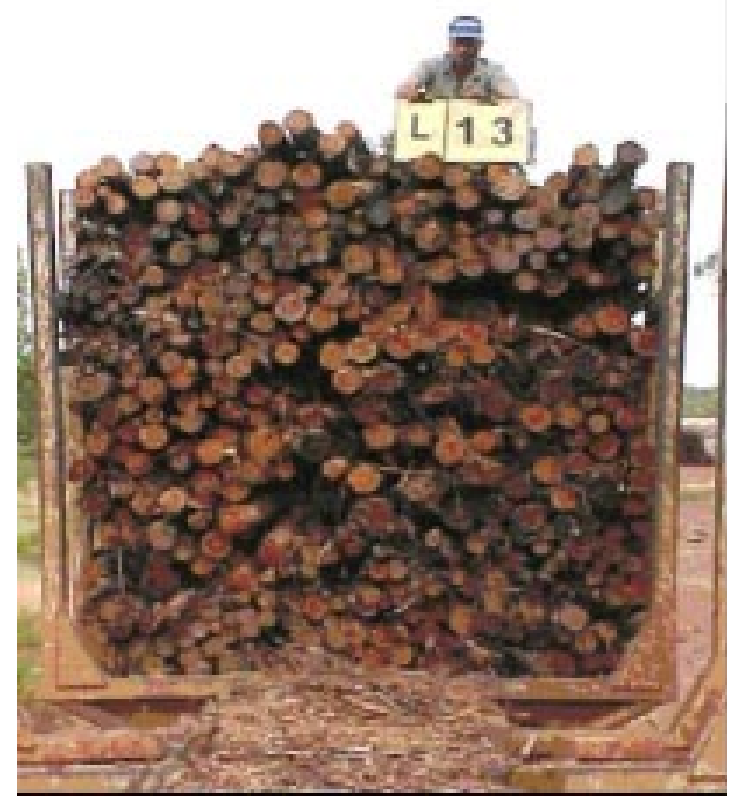

Figura 5 - Qualidade do empilhamento mecânico (caminhão 1- pilha 3).

Figure 5 - Mechanical piling quality (truck 1, pile 3).

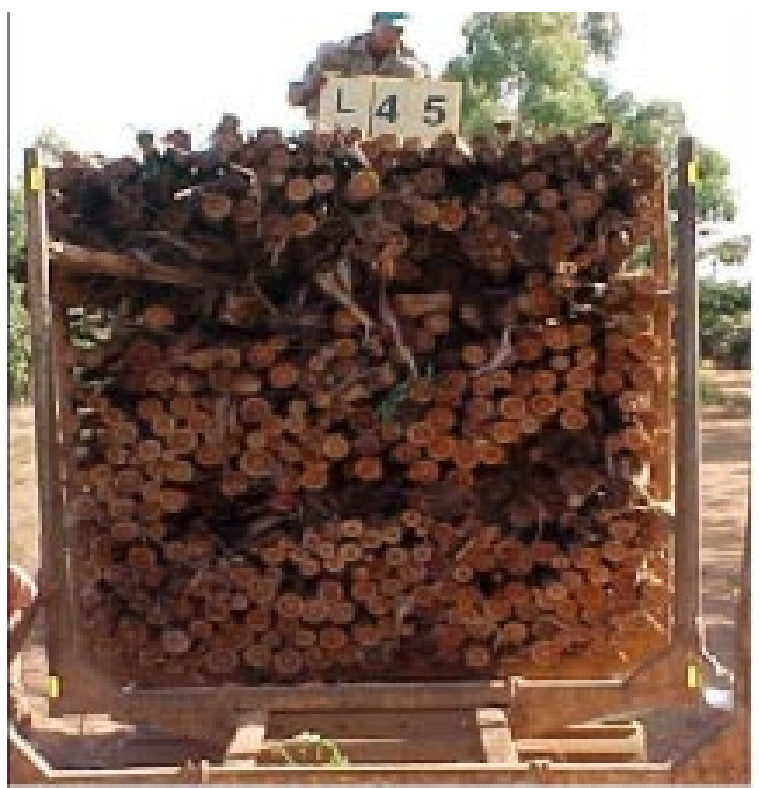

Figura 6 - Qualidade do empilhamento mecânico (caminhão 4 - pilha 5).

Figure 6 - Mechanical piling quality (truck 4, pile 5).
Quadro 3 - Fatores de empilhamento médios (seis repetições) para cada pilha e as diferenças porcentuais entre os fatores reais

Table 3 - Medium piling factors for each pile (six repetitions); and percentile differences between the actual factors

\begin{tabular}{|c|c|c|c|r|}
\hline $\begin{array}{c}\text { Caminhão } \\
\left(\mathrm{n}^{\mathrm{o}}\right)\end{array}$ & Pilha & $\begin{array}{c}\text { Fator } \\
\text { Real }\end{array}$ & $\begin{array}{c}\text { Fator Médio } \\
\text { Fotografia }\end{array}$ & $\begin{array}{c}\text { Diferença } \\
(\%)^{*}\end{array}$ \\
\hline & 1 & 0,5510 & 0,6150 & 11,6159 \\
& 2 & 0,5638 & 0,6060 & 7,4755 \\
$1(5221)$ & 3 & 0,5522 & 0,6041 & 9,3953 \\
& 4 & 0,5536 & 0,6013 & 8,6111 \\
& 5 & 0,5356 & 0,6103 & 13,9389 \\
\cline { 2 - 5 } & MÉDIA => & $\mathbf{0 , 5 5 1 3}$ & $\mathbf{0 , 6 0 7 3}$ & $\mathbf{1 0 , 2 0 7 3}$ \\
\hline \multirow{4}{*}{$4(5218)$} & 1 & 0,5891 & 0,6119 & 3,8787 \\
& 2 & 0,5746 & 0,5871 & 2,1697 \\
& 3 & 0,5556 & 0,6221 & 11,9603 \\
& 4 & 0,5509 & 0,6050 & 9,8248 \\
& 5 & 0,5413 & 0,6000 & 10,8367 \\
\cline { 2 - 5 } & Média $=>$ & $\mathbf{0 , 5 6 2 3}$ & $\mathbf{0 , 6 0 5 2}$ & $\mathbf{7 , 7 3 4 0}$ \\
\hline
\end{tabular}

* Diferença $(\%)=($ fotografia-real $) /$ real $* 100$.

Quadro 4 - Análise estatística das pilhas retirando-se os caminhões 1 e 4

Table 4 -Statistical analysis of the piles without trucks 1 and 4

\begin{tabular}{|c|c|c|c|}
\hline \multirow{2}{*}{$\begin{array}{c}\text { Pilha } \\
\text { No }\end{array}$} & \multicolumn{2}{|c|}{ Fator } & Diferença \\
\cline { 2 - 3 } Real-Fotografia
\end{tabular}


A título de exemplo, ao retirar as estimativas dos fatores dos caminhões 1 e 4 das análises, verificou-se que os fatores de empilhamento obtidos pelo procedimento fotográfico não diferiram dos valores reais, pelo teste "t" (Quadro 4). Este resultado indica que se o empilhamento for bem realizado o método fotográfico pode ser utilizado para determinação de fatores empilhamento com precisão $(<1 \%)$.

\section{CONCLUSÕES}

Entre as conclusões do presente trabalho, têm-se:

- A diferença porcentual média entre os fatores de empilhamento com casca obtidos pelo método fotográfico e os fatores de empilhamentos reais foi da ordem de 3,2\%, com tendência de superestimação dos fatores pelo método fotográfico.

- O mau empilhamento da madeira influenciou negativamente a precisão do método fotográfico, acarretando diferenças estatísticas entre os fatores de empilhamentos reais e os obtidos por fotografias.

- Fatores de empilhamento médios obtidos por repetições na mesma fotografia não acarretaram melhoria da precisão do método fotográfico.

\section{REFERÊNCIAS BIBLIOGRAFIAS}

CARVALHO, F. D. et al. Image processing system for the measurement of timber truck loads. In: INTERNATIONAL CONFERENCE ON MANUFACTURING AUTOMATION, Mentary, 1992, Hong Kong. Proceedings... Hong Kong: University of Hong Kong, 1992. p. 39-45.
GARLAND, H. Using polaroid camera to measure trucked hardwood pulpwood. Pulp Paper Canada, v. 69, n. 8, p. 86-87, 1968.

HUSCH, B.; MILLER, C. I.; BEERS, T. W. Forest mensuration. 2.ed. New York: John Willey \& Sons, 1972. $410 \mathrm{p}$.

KEEPERS, C. A. H. New method of measuring the actual volume of wood in stacks. Journal of Forestry, v. 43, p. 16-22, 1945.

LOETSCH, F.; ZÖHRER, F.; HALLER, K. E. Forest inventory. 2.ed. Munich: BVL Verlagsgesellschaft, 1973. v. 2, 469 p.

MOUTAIN, H. S. Determining the solid wood volume of four-foot pulpwood stacks. Journal of Forestry, v. 49, p. 627-631, 1949.

PAULA NETO, F.; REZENDE, A. V. Equações de fatores de empilhamento e tabelas de volumes em metro estere para árvores individuais. Revista Árvore, v. 16, n. 1, p. 72-87, 1992.

REZENDE, A. V. Análise dos métodos de estimação do volume sólido da madeira e do comportamento do fator de empilhamento para Eucalyptus grandis. 1988. $131 \mathrm{f}$. Dissertação (Mestrado em Ciência Florestal) - Universidade Federal de Viçosa, Viçosa, 1988.

RUBIO, M. E. L. Comparación de diferentes métodos para el calculo de coeficientes de apilamiento en bruzuelo, raya y troza de medidas comerciales. Mexico: Instituto Nacional de Invetigaciones Forestales, 1982. 64 p. (Boletim Técnico, 82). 